\title{
A Multimodal Discourse Analysis of Movie Posters From the Perspective of Visual Grammar — A Case Study of "Hi, Mom"
}

\author{
Zhuyan Peng \\ School of Foreign Languages, Chongqing Normal University, Chongqing, China
}

\begin{abstract}
Poster is one of the main modes of multimodal discourse and plays a very important role in film promotion. Based on the visual grammar system under the framework of meta-functional theory, this paper gives a multimodal discourse analysis on a poster of the movie $\mathrm{Hi}, \mathrm{Mom}$. The purpose is to interpret the reproducing meaning, interactive meaning and composition meaning of the film poster, which helps to explore the connotation and thematic meaning of the film poster discourse, so as to help people better understand the theme of the film.
\end{abstract}

Index Terms — visual grammar, multimodal discourse analysis, movie posters

\section{INTRODUCTION}

The wide application of multimedia technology makes multimodal means such as image and sound greatly enrich human communication activities. A discourse that expresses meaning does not just appear as a text pattern, multimodal discourses like images, colors and sounds also carry special meanings. Multimodal discourse analysis, which originated in the 1990s, has aroused great interest of scholars at home and abroad, especially functional linguists. They shifted the focus of the study on the social symbolism of language to images, voice, movement and other multimodal communication methods. Although the research of multimodal discourse in China is still in the initial stage, many scholars such as Li Zhanzi (2003), Hu Zhuanglin (2007), Zhu Yongsheng (2007) and Zhang Delu (2009) have made contributions to enrich and improve the theoretical framework of multimodal discourse analysis. These research achievements have greatly promoted the theoretical development and application of multimodal discourse analysis.

As the embodiment of a movie, a movie poster is a typical multimodal discourse integrated with image, text and color. It undertakes the task of highlighting the movie plot and attracting audiences' attention. In view of this, based on the theoretic framework of visual grammar from Kress and Van Leeuwen, this paper firstly starts with the theory of multimodal discourse and visual grammar, and then gives a systematic multimodal discourse analysis on an excellent poster of $H i$, Mom, which is a hit film in 2021.The purpose of this paper is to study how the film poster realizes the reproducing meaning, interactive meaning and composition meaning, so as to further interpret the implications of the poster and help people better understand the theme of the film.

\section{MultimODAL DisCOURSE}

Mode refers to the discourse mode perceived by information receivers, namely the channels and media of communication, including language, image, color, music and other symbol systems. Multimodal is a discourse form that combines images, sounds, tables and other communication modes in addition to words to convey information. In other words, multimodal discourse is the result of the comprehensive use of multiple modes in human communication. In short, multimodal discourse is a discourse composed of two or more single modes that express meaning.

Since the emergence of multimodal discourse analysis in the West in the 1990s, the theory of multimodal discourse analysis has developed rapidly and has attracted the attention of scholars at home and abroad. Multimodal discourse, which is derived from the linguistics research, is based on systematic functional linguistics founded by Halliday. Halliday (1978) believes that language, as a social symbol and a system of "meaningful potential", has three pure functions: the conceptual function of representing the objective world and the inner world, the interpersonal function of reflecting the communication participation and role relationship of language users and the textual function of organizing coherent discourse. Multimodal discourse scholars take this theory as a research perspective to extend the characteristics of social symbols in language to other symbol systems other than language, and believe that multimodal discourse is as versatile as language symbols, that is, with three meta-functions. Among them, the most representative one is the visual grammar proposed by Kress \& Van Leeuwen (1996). They believe that images are also social symbols and have the function of conveying significance. So they extend the idea of pure physical function to the visual mode and establish a framework of multimodal discourse analysis for image analysis. 
The visual grammar theory of multimodal discourse developed by Kress \& Van Leeuwen (1996) is based on the theory of systematic functional linguistics, in which the meta-function is proposed for language research, but not limited to language. In their study, they argue that the grammar system of language determines how words can be used to form clauses, sentences and discourse, while visual grammar determines how characters, things, etc. form visual statements with different degrees of complexity. Therefore, they extend the grammar of the language to the visual mode and put forward the three major meanings in the visual grammar corresponding to the representational meaning, interpersonal meaning and compositional meaning in the functional grammar. The construction of these three meanings can be said to be an innovative extension of meta-function ideas, providing a reliable theoretical perspective for the multimodal discourse analysis of images.

\section{A. Reproducing Meaning}

Reproducing meaning, corresponding to the conceptual meaning in the functional grammar, refers to the real reproduction of objective things and the relationship with the external world, which can be divided into narrative reproduction and conceptual reproduction. The difference between the two is whether the image representation is connected through a vector, in which the elements in the graph form oblique, usually strongly diagonal, forming the vector, which is the symbol of the narrative image. Among them, narrative reproduction includes the action process, the reaction process, and the speech as well as psychological process. During action process, the vector that sends out is the actor and the vector that is pointed serves as the goal. The reaction process is mainly the eye contact of the participant, that is, the vector is composed of eyes rather than action, so the participants in the reaction process become the reactor and phenomenon. The vectors in speech and psychological processes are mainly connected by the participants and the thinking bubble or the dialogue bubble: the content of the thinking bubble is indirectly presented through the sensor, while the content of the dialogue bubble is reflected in the through the speaker. Conceptual reproduction includes classification, analytical, and symbolic processes. The analysis and classification processes focus on the study by which relationships the participants are interconnected, while the symbolic processes study what the participants are, which actually correspond to the relational processes and existence processes in the conceptual meaning.

\section{B. Interactive Meaning}

Interactive meaning, corresponding to the interpersonal meaning in the functional grammar, aims to explore the particular social relationship between the image producer and viewer. Images can cause specific relationships between the viewer and the world in the image. They interact with the viewer and prompt the viewer's attitude towards the reproduced scene. The realization of interactive meaning mainly depends on four elements: contact, attitude, and modality. They interact together to create a complex and delicate relationship between the viewer and the reproduced content.

Contact reflects some imaginary relationship between the viewer through the eye contact of the participant in the image, which is composed of request and provision. If participants look to the viewer, it will form request; while provision is just passing the message rather than pointing to the audience. Social distance determines the affinity of the image participant and the viewer, usually related to the frame size of the shot. The smaller the lens frame, the closer the relationship and vice versa. Attitude refers to the subjective opinion of the viewer to the participant, mainly reflected by the perspective. Different perspectives reflect different attitudes. From the horizontal perspective, the front-view direction transmits a sense of immersion and empathy and side direction shows the viewer's indifference. From the vertical perspective, overlooking shows the strength of the viewer, looking up represents the participant in the strong position and parallel angle shows the equality. Modality refers to the truth and credibility of the statements people make to the world they concern. Halliday (1978) divides the modality into high, middle and low levels. Kress $\&$ van Leeuwen (1996) explore the high and low of the magnitude of modality in an image from eight visual markers, that is, color saturation, color discrimination, color coordination, contextualization, performance, depth, illumination, and luminance of light.

\section{Composition Meaning}

Compositional meaning, corresponding to the textual meaning in the functional grammar, refers to how the image integrates reproducing meaning and interactive meaning to form a meaningful whole. It mainly includes information value, viewfinder, and significance. The information value is represented by the location of the different components in the overall composition, and the role of any particular element in the ensemble depends on whether it is placed on the left or right, top or bottom. The composition from top to bottom can convey ideal to real structure, and the composition from left to right can establish the structure from known to new information. The so-called viewfinder is the size of the composition component, by using the architectural method to create the lines of the actual segmentation framework to cut off or connect the elements in the image, to symbolize whether they belong to each other in some aspects. Significance is reflected in the different degrees to which the elements attract the attention of the viewer, and can be achieved by the position where people or objects are placed, relative size, color value contrast (or color), brightness, etc.

\section{An Analysis of Movie Posters From the Perspective of the Visual Grammar}


In this poster, the images, colors, text and other symbols complement each other and participate together in the construction of the film theme. As a kind of graphic visual art, film posters often take pictures as the main form of communication. The picture is foregrounding, and various social symbols such as language, color and composition in it are coordinated with the role of supplementing and strengthening the theme of the film. Then this poster will be analyzed from three aspects of the Reproducing meaning, interactive meaning and composition meaning.

\section{A. Reproducing Meaning in the Film Poster}

Kress \& van Leeuwen (1996) distinguish the reproducing meaning of the image into narrative and conceptual categories. They believe that the narrative images are an interpretation of phenomenon, while the conceptual images are reflecting the essence. The poster consists of two images, both mainly narrative images. The mother and baby in the front end of the picture are the most prominent participants, which occupy the largest poster size and have sufficient light. Compared with the hazy characters in the background, the overall color of the picture is relatively bright, with a clear focus and a real sense. The gaze of the mother and infant constitutes the vector of this picture, and therefore it is a narrative image representing the reaction process. The mother's eyes are slightly down, showing a tenderness and love. The baby's eyes are up, showing a trace of joy and loveliness. In this process, it can be said that mother is the responder because the baby her view pointing to is the object of perception, or can be said that the baby is the responder and the mother is the perceived phenomenon. When participants are connected by a vector and interact with each other, representing they are doing something to each other, which reflects the mutual eye contact. In the back part of the picture, standing outside by the window is another hostess of the film. We can see her vision looking slightly down, with a little sadness and comfort, looking at such warm frame, focusing on the smiling mother, which forms the vector. At this time, as a participant, she was a lonely responder, and the direction of the gaze was the orientation of the vector, that is, the mother is a phenomenon. However, the mother did not form a convective eye interaction with her. At this time, the poster image shows the reproducing meaning of the narrative, creating a warm and sentimental atmosphere, and we can feel the daughter's deep yearning for the mother.

\section{B. Interactive Meaning in the Film Poster}

Kress \& Leeuwen (1996) believe that interactive meaning is about the relationship between the image producer and the viewer and it prompts the viewer's attitude towards representing things, which consists of four elements: contact, social distance, attitude and affection.

First is contact. Contact is divided into request and provision, meaning an imaginary contact relationship established between the participant and the viewer through the direction of the gaze. None of the characters in the poster looks directly at the audience and there is no connection with the viewer, as if they were the specimens displayed there, providing information through their facial expressions and posture. Therefore, the poster is a picture of provision responsible for providing the meaning of the image information. As mentioned before, the mother's slightly downward eyes reveal a tenderness and love, and the baby's slightly upward eyes just meet her mother. At this time, the daughter's vision in the back part and her mother's vision form a right Angle. From her eyes, it seems to see a trace of sadness and happiness. The posture and scene of the mother holding the bowl and the baby with her spoon show a simple and happy sense of warmth. In sharp contrast to her daughter, who stands alone outside the window, the warm atmosphere contains a kind of sadness.

Second is social distance. Social distance is reflected by the shooting, and the distance of the camera reflects the interpersonal relationship between the participants and the viewer. In the two images of the poster, the distance between the characters and the audience is different. Close-up suggests intimate relationships, while long shots suggest distant relationships. In the first part of the picture, the mother and baby waist above take a relatively large room, belonging to personal distance. We can feel the proper distance between the participants and the audience, neither close or far away. This distance allows the audience to feel the intimate emotion between the mother and the baby, but this emotion is only between two people, forming a certain boundary with the audience. The latter part takes a public distance, with the daughter standing outside the window being surrounded by the surrounding space, about the size of three or four people. The daughter in the picture is looking at her mother, feeling different from the time and space her mother is in. And the audiences observe the daughter in the form of the attitude of an onlooker. Such a scene opened the distance from the audience and excluded the audience, which can only be quietly seen in the distance, making us objectively think about the daughter's heart and fate.

Third is attitude. Attitude is reflected through the "perspective" to express the subjective and objective attitude of the viewer. From a horizontal dimension, the poster adopts a positive perspective, making the audience feel empathy and naturally integrate into it and resonate. From the vertical dimension, the mother looks down on the baby while the infant looks up at the mother, indicating that one is on the strong status and the other is weak, that is, the mother is the guardian of the baby and the infant is the object of protection. The daughter looks parallel at the mother and tends to convey an objective message, which shows an equal relationship. Based on the two perspectives, we can not only intervene in the world of mother and baby and be moved by their warm scenes, but also feel the mood of the daughter from the identity and perspective of her peers.

The last one is modality. It refers to the degree of expression method used in image analysis. The three levels of modality are distinguished from color saturation, color differentiation, color coordination, contextualization, 
performance, depth, lighting, and brightness. Color is the most common visual element of movie posters. Most of the poster uses light yellow, tender pink, green and other moderate saturated colors to create a warm scene atmosphere. With a very peaceful appeal, there seems to be a quiet time to tell the story, causing the emotional resonance of the audience. The latter part of the picture mainly uses lower saturation colors such as beige, forming a feeling of time and space union and separation, which also suggests that the daughter's ending in trying to reverse her mother's fate will only fail. On the whole, the poster does not have a great color distinction and the color coordination is relatively stable. The whole is relatively bright, presenting a warm and soft tone, which belongs to the middle modality. This will not cause a great visual impact to the audience and bring people a real and credible feeling, making people feel calm and comfort.

In terms of contextualization and performance, this poster simply reproduces the scene of mother and baby eating in the family. The objects around it are also simple decorations. The daughter outside the window is not clearly shown in front of the audience. It is obviously very close but seems to be very far away, giving people a very real feeling. In depth, this poster is also characterized by neutral modality. The prospect of the image is part of a relatively closed room, with mothers, babies and furnishings occupying a large space, making the audience feel a sense of happiness in an ordinary life. The back scene is a slightly wider space, in which stands a slightly blurred daughter, giving the audience a distant and sentimental feeling.

\section{Composition Meaning in the Film Poster}

Composition meaning correlates the elements in the different meanings of the image through the information value, viewfinder, and significance. First of all, in terms of information value, the poster's mother and the baby and some of the specific furnishings are under the picture, which is "real". The information makes people feel real and credible and the scene created also gives people a kind of natural and loving happiness. Above the picture is the background of the daughter and some hidden branches outside the window, which is "ideal". It implies that the existence of the daughter in the film at this time is nothingness, and the thoughts and actions of trying to change the mother's life track will be just an "ideal", an impossible idea, which is the collision of "real" and "ideal". The mother smoothed her daughter's inner guilt with practical experience and helped her daughter get out of the shadow of the bottom of her heart, and finally realized her daughter's "ideal" from another Angle. From a viewfinder perspective, the poster takes two scenes, which is divided by the obvious bezel line around the window. The image of the mother and the baby accounts for almost three-quarters of the picture, and a small part consists of the daughter and the location, which very cleverly connects the mother to the "future" daughter. Regarding significance, the most striking part of the film posters is the mothers and baby. One of the most prominent parts is the mother's gentle and friendly expression, shining light yellow dress and the posture of bowl holding, which is in line with the name of the film and better fit with the theme of the film. Then is the daughter outside the window. Although her overall size is not large, but the comparison between her and her mother makes people cannot ignore her existence. It can not only reflect her daughter's sad and gratified mood, but also achieve the effect of publicity to attract the audience.

\section{CONCLUSION}

This paper analyzes the multimodal discourse based on the theoretical framework of visual grammar combined with examples. In the discourse mode of the movie poster, the picture, color, and other symbols in the image together participate in the construction of the film theme. It is the synergistic effect of these symbols to fully reflect the overall meaning of the film discourse, convey the theme and plot of the film and attract interest of the audiences. The analysis of images from the perspective of visual grammar starts from the three functional meanings of reproduction, interaction and composition, paying attention to the internal characterization factors and structure of the discourse, which has a good explanatory power for it. At the same time, it is also helpful to comprehensively understand the story, explore the deeper significance of the film posters, and also improve the audience's film appreciation ability. Therefore, as a common multimodal expression mode in the film propaganda, it is important to analyze and study the posters from the visual grammar theory.

\section{REFERENCES}

[1] Halliday, M.A.K. (1978). Language as a Social Semiotic: The Social Interpretation of Language and Meaning, London: Edward Arnold.

[2] Hu, Zh. L. (2007). She hui fu hao xue zhong de duo mo tai hua [Multimodality in social semiotics studies]. Language Teaching and Research, $(01): 1-10$.

[3] Kress, G. \& Van Leeuwen, T. (1996). Reading Images: The Grammar of Visual Design, London: Routledge.

[4] Li, Zh. Z. (2003) Duo mo tai hua yu de she hui fu hao xue fen xi. [Social semiotic analysis of multimodal discourse]. Foreign Language Studies, (05): 1-8 + 80 .

[5] Tian, L \& Liu, Z. Q. (2008). She hui fu hao xue shi jiao xia de duo mo tai hua yu fen xi-yi shang hai shi bo hui hui hui wei li. [Multimodal discourse analysis from a Social Symbolic perspective - a case study of the Shanghai World Expo]. Journal of Yanshan University (Philosophy and Social Sciences Edition), (03): 123-126.

[6] Wang, J. H. (2019). Zheng wu xin mei ti zhao pian hua yu de shi jue yu fa -yu yong fen xi. [Visual grammar of government new media photo discourse-language analysis]. Contemporary rhetoric, (02): 72-83. 
[7] Xu, Y. W \& Xiao, T. T. (2021). Shi jue yu fa shi jiao xia cheng shi xuan chuan pian de duo mo tai hua yu fen xi. [Multimodal discourse analysis of urban propaganda videos from a visual grammar perspective]. Journal of Harbin College, 42 (04): 104-106.

[8] Yuan, Y. Y \& Zhang, D. L. (2012). Duo mo tai dian ying hai bao yu pian de she hui fu hao xue fen xi. [Social semiotics analysis of the multimodal movie poster discourse]. Journal of Jining University, 33 (02): 35-39.

[9] Zhang, D. L. (2009). Duo mo tai hua yu fen xi zong he li lun kuang jia tan suo [Comprehensive theoretical framework of multimodal discourse analysis]. Chinese Foreign Language, 6 (01): 24-30.

[10] Zhang, J.Y. \& Jia. P. P. (2012). Guan yu shi jue yu fa de ji dian si kao. [Several reflections on the visual grammar]. Contemporary Foreign Language Studies, (03): 38-42 + 160 .

[11] Zhu, Y. Sh. (2007). Duo mo tai hua yu fen xi de li lun ji chu yu yan jiu fang fa [Theoretical basis and research method of Multimodal discourse Analysis]. Foreign Language Journal, (05): 82-86.

Zhuyan Peng was born in Danyang, China in 1998. She received her Bachelor's degree in English education from Yichun University, Jiangxi, China in 2020.

She is currently a postgraduate student in the School of Foreign Languages, Chongqing Normal University, Chongqing, China. Her research interests include language teaching and linguistics. 